\title{
Psikolojik İyi Oluş: Zaman Perspektifi ve Psikolojik Esnekliğin Rolü
}

\author{
Lütfiye Nur Akkoç Arabac1 ${ }^{1}$ \\ İstanbul Medipol Üniversitesi
}

\author{
Gizem Cesur Soysal ${ }^{2}$ \\ İstanbul Medipol Üniversitesi
}

\section{Özet}

Mevcut çalışmada zaman perspektifi ve psikolojik iyi oluş ilişkisi ve bu bağlamda psikolojik esnekliğin rolünün incelenmesi amaçlanmaktadır. Araştırmaya 18 yaş üstü 350 kişi katılmıştır. Katılımcıların yaş ortalaması 29.53’tür ( $s s=$ 6.95). Çalışmada veri toplamak amacı ile Demografik Bilgi Formu, Zimbardo Zaman Perspektifi Envanteri, Psikolojik İyi Oluş Ölçeği, Kabul ve Eylem Formu-II kullanılmıştır. Elde edilen bulgular ilk olarak zaman perspektifinin beş alt boyutunun ve psikolojik katılığın psikolojik iyi oluşu anlamlı düzeyde yordadığını ortaya koymuştur. Ardından psikolojik katılık düzeyinin aracı rolünü test etmek amacıyla Hayes (2013) tarafından geliştirilmiş olan PROCESS makro eklentisi kullanılmıştır. Buna göre dengeli zaman perspektifi sapma puanının psikolojik iyi oluş üzerinde psikolojik katılık aracılığıyla dolaylı etkisinin anlamlı olduğu görülmüştür. Bu çalışma ile psikolojik iyi oluş üzerinde dengeli zaman perspektifi ve psikolojik esnekliğin birlikte ele alınmasının önemi ortaya konmuştur. Bulgular gerek klinik müdahaleler gerekse ileriki araştırmalar için uzmanlara yeni bir bakış açısı sunabilecek niteliktedir.

Anahtar kelimeler: Zaman perspektifi, dengeli zaman perspektifi, psikolojik iyi oluş, psikolojik esneklik, psikolojik kat1lik

\begin{abstract}
The main purpose of this study is to investigate the role of psychological flexibility on the relation between time perspective and psychological well-being. The sample consisted of 350 people who are 18 years of age and over. The average age was $29.53(S D=6.95)$ years. Socio-demographic Information Form, Zimbardo Time Perspective Inventory, Flourishing Scale and Acceptance and Action Questionnaire-II were used to collect data. First, the results indicated that time perspectives profiles and psychological inflexibility significantly predicted psychological well-being. Second, the PROCESS macro plug-in developed by Hayes (2013) was conducted to test the mediating role of the psychological inflexibility level. Accordingly, it was observed that the indirect effect of deviations from a balanced time perspective on well-being through psychological inflexibility was significant. Consequently, this study indicates the effect of balanced time perspective and psychological flexibility together on psychological well-being. The findings are capable of providing new insight to experts for both clinical interventions and future research.
\end{abstract}

Keywords: Time perspective, balanced time perspective, psychological well-being, psychological flexibility, psychological inflexibility

Yazışma Adresi: ' ${ }^{M}$ aster Öğr. Lütfiye Nur Akkoç Arabacı, İstanbul Medipol Üniversitesi, İnsan ve Toplum Bilimleri Fakültesi, Psikoloji Bölümü, Kavacık Yerleşkesi, Beykoz / İstanbul, 1.nur.akkoc@gmail.com, ORC-ID: 0000-0001-7513-3383

${ }^{2}$ Öğr. Gör. Dr. Gizem Cesur Soysal, İstanbul Medipol Üniversitesi, İnsan ve Toplum Bilimleri Fakültesi, Psikoloji Bölümü, Kavacık Yerleşkesi, Beykoz / İstanbul, gizemcsr@gmail.com, ORC-ID: 0000-0001-8025-6927

Gönderim Tarihi: 30.07 .2020

Kabul Tarihi: 12.07 .2021 
Zaman kavramı tarihsel süreçte farklı disiplinler içinde her zaman merak edilen ve araştırılan bir konu olmuştur (Babür, 2014; Buccheri, Gesù ve Saniga, 2012; Lewin, 1951). Psikoloji biliminin zamanı ele alışı ise yakın bir geçmişe sahiptir. İlk kez zaman ve insan psikolojisi hakkında fikir ve düşüncelerini sunan bilim insanlarından olan William James (1890) zamanın sadece fiziksel bir fenomen olmadığını aynı zamanda psikolojik bir değerlendirme de olduğunu söylemiştir. Daha güncel olarak ele alınabilecek olan Zimbardo ve Boyd'un (1999) zaman kavramı ise Lewin'in (1951) "zamansal bakış açısını" tanımladığı; kişinin "andaki”" gelecek ve geçmiş hakkında oluşturduğu psikolojik bakış açılarının bütünü görüşüne dayanmaktadır. Zimbardo ve Boyd (1999) zaman kavramı içerisinde spesifik olarak Lewinian bir gelenekle "zaman perspektifi” üzerine çalıșmışlardır. Buna göre zaman perspektifi bireyin deneyimlediği yaşam akışını geçmiş, şimdi ve gelecek olarak zamansal bir kategoriye ayırması olarak tanımlanmıştır (Zimbardo, Keough ve Boyd, 1997). Yapılan araştırmalar bu üç zaman dilimine odaklanmanın bireysel farklılıklar ve psikolojik faktörler ile ilişkisini ortaya koymaya devam etmektedir (Laghi, Baiocco, D'Alessio ve Gurrieri, 2009; Strathman, Gleicher, Boninger ve Edwards, 1994; Zhang ve Howell, 2011). Örneğin gelecek zaman yönelimi ile psikopati arasında negatif, fiziksel sağlık için yapılan davranışlar (kanser taramasına katılma, diş ipi kullanma, prezervatif kullanma) ve akademik başarı arasında pozitif yönde ilişki bulunmuştur (Zimbardo ve Boyd, 1999).

Zimbardo ve Boyd (2018) kişinin belirli bir zaman perspektifi oluşturmasını yaşamsal deneyimler, kültür, eğitim, sosyal sınıf, din, yaşanılan coğrafya, iklim gibi etmenler ile öğrenilen yargılara dayandırmaktadırlar. Rutin şekilde kullanılan zaman perspektifi alışkanlık haline gelir ve süreklilik gösterir. Böylece kişi genelde fark1nda olmadan zaman perspektiflerinden birini diğerine tercih etmektedir. Sürekli kullanılan bu zaman perspektifi ise kişinin duygu, düşünce ve davranışları üzerinde önemli rol oynayan en güçlü etkenlerden biridir (Holman ve Zimbardo, 2009). Zimbardo ve Boyd (1999) zaman perspektifini "olumlu geçmiş", "olumsuz geçmiş", "hazc1 şimdi", "kaderci şimdi" ve "gelecek" yönelimi olmak üzere beş boyutta ele almıştır.

Olumlu geçmiş zaman perspektifi kişinin geçmişine ait tutumunu duygusal, nostaljik ve hoş olarak temsil ederken aile ve arkadaşlarla ilişkilerin sürdürülmesini vurgular. Yapılan araştırmalarda bu perspektiften alınan yüksek puanın depresyon, öfke ve kaygıyı negatif, özsaygıyı pozitif yordadığı gözlemlenmiştir (Zimbardo ve Boyd, 1999). Ayrıca bu zaman diliminin daha geniş sosyal çevre ve daha uzun süreli ilişki kurma ile de pozitif ilişki içinde olduğu görülmüştür (Holman ve Zimbardo,
2009). Olumsuz geçmiş zaman perspektifi kişinin geçmişini negatif ve rahatsız edici olarak değerlendirmesidir. Travmatik ve olumsuz olaylar bu değerlendirmeye sebep olabilirken, sadece kişinin bakış açısı ya da iki durumun etkileşimi bu yönelimi ortaya çıkarabilir (Zimbardo ve Boyd, 1999).

Hazcı şimdi zaman perspektifi kişinin andaki hazza odaklandığı ve sonrasında elde edeceği sonuçlarla ilgilenmediği tutumdur. Yapılan araştırmalar sonucunda hazcı şimdi yönelim ile pozitif mood (Stolarski, Matthews, Postek, Zimbardo ve Bitner, 2014) ve öznel iyi oluş arasında pozitif ilişki gözlemlenmiştir (Drake, Duncan, Sutherland, Abernethy ve Henry, 2008). Kaderci şimdi zaman perspektifi bireyin şimdide bulunacağ ranışlar ile geleceği şekillendiremeyeceği ve geleceğin tahmin edilemeyeceğine dair olan tutumudur. Zimbardo ve Boyd (1999) bu tutuma sahip kişilerin depresyon, kayg1 ve öfkede yüksek puanlara sahip olduğunu göstermektedir. Ayrıca araştırmalarda kaderci şimdi zaman perspektifi ile psikopatoloji arasında pozitif (Van Beek, Berghuis, Kerkhof ve Beekman, 2011), iyi oluş arasında negatif ilişki olduğu ortaya konmuştur (Seema ve Sircova, 2013).

Gelecek zaman perspektifi ise gerçekleştirilecek hedefleri ve başarıyı dikkate alarak şu anki zamanda planlı hareket etmeyi tanımlar. Yapılan araştırmalarda gelecek zaman yönelimi ile psikolojik iyi oluş (Sailer ve ark., 2014) ve yaşam doyumu (Boniwell, Osin, Alex Linley ve Ivanchenko, 2010) arasında pozitif ilişki olduğu ortaya konmuştur.

İnsan davranışı üzerinde güçlü etkisi olan zaman perspektifinin değerlere ve ihtiyaçlara göre şekillenen esnek ve en uygun şekilde kullanımı üzerine yapılmış olan araştırmalara da rastlanmaktadır. Dengeli zaman perspektifi (DZP) olarak isimlendirilen bu kavram, içinde bulunulan durumun gerektirdiklerine ve kişisel kaynaklara bağlı olarak zaman perspektifleri arasında etkili bir zihinsel geçiş yapma yeteneği olarak tanımlanmaktadir (Zimbardo ve Boyd, 1999). DZP zaman perspektifi alt boyutlarından olumlu geçmiş puanın yüksek, gelecek ve hazcı şimdi puanlarının orta-yüksek, olumsuz geçmiş ve kaderci şimdi zaman perspektifi puanlarının düşük olmasidir (Boniwell ve Zimbardo, 2015). Zaman perspektifleri arasında esnek geçişler yapabilmek DZP'nin en önemli bileşenlerinden biri olmakla birlikte (Boniwell, 2005) DZP iyi oluş üzerinde önemli rol oynamaktadır (Boniwell ve Zimbardo, 2015).

Psikolojik iyi oluş pozitif-negatif duygular arasındaki ayrım ve yaşam doyumu temelinde tanımlanabilir (Andrews, 1974; Bryant ve Veroff, 1982; Stock, Okun ve Benin, 1986). Duygular ve yaşam doyumu psikolojik iyi oluşun merkezi kabul edilmektedir (Ryff, 1989). Psikolojik iyi oluş insan gelişimi ve varoluşsal zorlukla- 
rın çözümlenmesine odaklanmaktadır (Keyes, Shmotkin ve Ryff, 2002). Yapılan araştırmalar psikolojik iyi oluş puanının yüksek olmasının sadece iyi hissetmekle ilişkili değil aynı zamanda daha istikrarlı evlilikler (Kim ve McKenry, 2002), yüksek gelir (Kaplan, Shema ve Leite, 2008), daha yaratıcı fikirler (Ghorbani ve Kazemi-zahrani, 2015) ve daha güçlü bağışıklık sistemi ile ilişki halinde olduğunu göstermektedir (Lyubomirsky, King ve Diener, 2005).

DZP ile psikolojik iyi oluş arasında yapılan çalışmalar bu iki kavramın pozitif yönlü bir ilişki içinde olduğunu göstermektedir (Jankowski, Zajenkowski ve Stolarski, 2020; McKay ve ark., 2019; Webster ve Ma, 2013). DZP ile iyi oluş ve bilgelik arasındaki ilişkiyi inceleyen bir çalışmada bu üç kavramın birbiri ile güçlü bir ilişki içinde olduğu bulunmuştur (Webster, Bohlmeijer ve Westerhof, 2014). Bununla beraber DZP'ye ulaşmak için zaman perspektifleri arasında esnek geçişlerin önemi de dikkat çekmektedir. Bu temelde psikolojik esnekliğin DZP ve psikolojik iyi oluş arasında aracı role sahip olabileceği düşünülmektedir.

Psikolojik esneklik\katılık kavramı Kabul ve Kararlilık Terapisi (Acceptance and Commitment Therapy [ACT]) temelinde ele alındığında (Hayes, Strosahl ve Wilson, 1999), şimdiki anla temas halinde olmak, duyguların, duyumların ve düşüncelerin hoşa gitmeyenler de olsa farkında olmak, onları karşılamak ve kişisel değerler doğrultusunda hareket etmek olarak tanımlanır (Ramaci, Bellini, Presti ve Santisi, 2019). ACT davranışsal repertuvarın daralmasını psikolojik katılık olarak isimlendirmekte ve bunu psikopatoloji ile eş anlamda kullanmaktadır (Hayes, Luoma, Bond, Masuda ve Lillis, 2006). Repertuar darlığ1, pozitif ve negatif pekişme süreçleri ile belirli bir uyarana karşı belirli bir davranışın ortaya çıkmasıdır. Eğer bu davranış kişinin uzun vadeli hedefleri ve değerleri ile uyumsuzsa kişinin işlevselliğine zarar verir (Yavuz, 2015). ACT'in temel amacı kişinin psikolojik esnekliğini arttırmak, davranış repertuarını genişletmek (Luoma ve Vilardaga, 2013) ve bu şekilde zengin, dolu ve anlamlı bir yaşam oluşturmaktır (Harris, 2018). Yapılan araştırmalarda kaçınma ve psikolojik katılığa müdahale ederek esnekliği arttıran ACT'in anlamlı klinik sonuçlar elde ettiği görülmüştür (Hayes, Luoma, Bond, Masuda ve Lillis, 2006). Bir meta analiz çalışmasında da ACT'in anksiyete, depresyon ve travma sonrası stres bozukluğu gibi çeşitli klinik bozuklukların tedavisinde etkili olduğu ve kısa bir geçmişi olan ACT'in umut vadeden bir yaklaşım olduğu sonucuna varılmıştır (Powers, Zum Vörde Sive Vörding ve Emmelkamp, 2009). Üniversite öğrencilerinin iyi oluşlarını yükseltmek amacıyla uygulanan beş haftalık çevrimiçi ACT seanslarının ise katılımcıların iyi oluşlarını, yaşam doyumlarını ve farkındalık yeteneklerini yükselttiği göz- lemlenmiştir (Räsänen, Lappalainen, Muotka, Tolvanen ve Lappalainen, 2016). Yapılan bir diğer çalışmada ise ACT müdahaleleri ile psikolojik esnekliği yükseltmenin stres seviyesinin düşmesi ile negatif, psikolojik iyi oluşun yükselmesi ile pozitif ilişkili olduğu görülmüştür (Wersebe, Lieb, Meyer, Hofer ve Gloster, 2018).

\section{Mevcut Çalışma}

Mevcut çalışmanın amacı psikolojik iyi oluş düzeyi üzerinde zaman perspektifi ve psikolojik esnekliğin yordayıcılığını araştırmaktır. İkinci olarak DZP ve psikolojik iyi oluş ilişkisi bağlamında psikolojik esnekliğin aracı rolünü incelemektir. Yurtdışında yapılan çalışmalar incelendiğinde kültür ve coğrafyaya özgü zaman perspektifi profillerinin çıkarıldığ 1 ve bunun üzerine incelemeler yapıldığı görülmektedir (Sircova ve ark., 2014). Öğrenilmiş bir süreç olan zaman perspektifi kültürden bağımsız ele alınamayacağı için ülkemizdeki çalışma eksikliği dikkat çekmiştir. Mevcut çalışmanın bu temelde alanyazına katkısı olacağı düşünülmektedir. Bunun yanı sıra, dengeli ve ideal zaman perspektifinin tanımında profiller arasında esnek geçişler yapabilmenin önemli olduğu vurgulanmaktadır (Boniwell, 2005). Esnekliğin değerinin sik sik vurgulandığı zaman perspektifi alanında, psikolojik esnekliğin nasıl bir rolü olduğuna dair yapılmış herhangi bir çalışmaya rastlanmamıștır. Dolayısıyla psikolojik esneklik kavramının zaman perspektifi ile olan ilişkisini incelemenin bu alana özgün bir katk1 sunacağı düşünülmektedir. Bu kavramların birlikte ele alınmasının psikolojik iyi oluş düzeyi ile olan ilişkisinin ortaya konması ise iyi oluş alanyazınına yeni bir bakış açısı kazandırabilir niteliktedir. Aynı zamanda elde edilen sonuçların gerek araştırma gerekse uygulama alanına değerli katkılar sunabileceği düşünülmektedir.

\section{Yöntem}

\section{Katılımcılar}

Araştırmaya 18 yaş üstü 353 kişi katılmıştır. Örneklem sayısı Green'in (1991) regresyon analizleri için önerdiği $\mathrm{N}>50+8 \mathrm{~m}$ ( $\mathrm{m}$; araştırmadaki bağımsız değişken sayısı) formülü referans alınarak hesaplanmıştır. Araştırmanın örneklemi, araştırmacıya zaman ve maliyet açısından kolaylık sağlayan rastgele örneklem yöntemi ile elde edilmiştir

Çalışmaya katılan kişilerin $(\mathrm{N}=350)$ 144'ü kadın (\%41.1), 206's1 erkektir (\%58.9). Katılımcıların yaşları 18 ile 48 arasında değişmektedir ve yaş ortalaması 29.53 $(S s=6.95)$ olarak belirlenmiştir. Eğitim düzeyleri açısından incelendiğinde katılımcıların 27'sinin (\%7.7) lise ve alt1, 244'ünün üniversite (\%69.7), 79'unun (\%22.6) yüksek lisans derecesine sahip olduğu görülmektedir. Katılımcıların sosyo-ekonomik düzeylerine ilişkin veri 
elde etmek amaciyla sorulan soruya verilen yanitlardan, katılımciların 70'inin (\%20) alt ve orta alt, 189'unun (\%54) orta, 91'inin (\%26) orta ve orta üst gelir grubunda olduğu gözlemlenmektedir. Çalışma durumları incelendiğinde ise katılımcıların 207'si (\%59.1) bir işte çalış1yor olduklarını belirtmiştir.

\section{Veri Toplama Araçları}

Demografik Bilgi Formu: Araştırmaya katılan bireylerin demografik bilgileri hakkında bilgi toplamak amacıyla araştırmacı tarafından hazırlanmış anket sorularını içermektedir.

Zimbardo Zaman Perspektifi Envanteri (ZZPE): Katılımcıların zaman perspektiflerini değerlendirmek amaciyla Zimbardo Zaman Perspektifi Envanteri kullanılmıştır. Zimbardo ve Boyd (1999) tarafından geliștirilen anket 56 sorudan ve beş alt boyuttan oluşmaktadır. Ölçek 5'li likert tipindedir. Alınan yüksek puanlar zaman yönelimindeki farklılıkları ölçmektedir. Ölçeğin Türkçe geçerlilik güvenirlik çalışması Akırmak (2019) tarafından yapılmış ve alt boyutlara ilişkin Cronbach alfa katsayısının .68 ile .84 arasında olduğu görülmüştür. Mevcut çalışma için Cronbach alfa tutarlılık katsayıları ise .87 ve .71 arasında değişmektedir. Ayrıca çalışmada zaman perspektifi alt boyutları ile birlikte dengeli zaman perspektifi sapma puanı kullanılmıştır. Dengeli zaman perspektifi sapma puanı kişinin ideal zaman perspektifine ne kadar uzak olduğunu göstermektedir. Stolarski ve arkadaşları (2011) tarafından kültürler arası çalışmalar temel alınarak ideal sapma puanları olumlu geçmiş zaman için 4.60; olumsuz geçmiş zaman için 1.95; hazc1 şimdi için 3.90; kaderci şimdi için 1.50; gelecek yönelimi için 4; şeklinde belirlenmiştir. Sapma puanları ile aşağıdaki formül kullanılarak dengeli zaman perspektifi sapma puanı (DZSP) elde edilmiştir.

$\mathrm{DZSP}=\sqrt{\begin{array}{c}(i N G-e N G)^{2}+(i P G-e P G)^{2}+(i S ̧ K-e S ̧ K)^{2}+ \\ (i S ̧ H-e S ̧ H)^{2}+(i G-e G)^{2}\end{array}}$

$i=$ ideal sapma puanı, $e=$ kişiden Zimbardo Zaman Perspektifi Envanteri ile elde edilen puan

$N G=$ olumsuz geçmiş, $P G=$ olumlu geçmiş, $S$ K $=$ şimdi kaderci, $S$ H $=$ şimdi hazcl, $G=$ gelecek

Psikolojik İyi Oluş Ölçeği (PİÖ): Diener ve arkadaşları tarafından (2009) geliştirilen psikolojik iyi oluş ölçeği anlamlı bir yaşam, pozitif ilişkiler, yeterli kendilik algısı, bir amaca sahip olmak gibi temaları içeren 7'li likert tipi ile derecelendirilmiş 8 maddeden oluşmaktadır. Ölçekten alınan yüksek puan kişinin psikolojik iyi oluşunun yüksek olduğunu göstermektedir (Diener ve ark., 2009). Telef (2013) tarafından Türk kültürüne uyarlama çalışması yapılmış ve Cronbach alfa tutarlık katsayısı .80 olarak belirlenmiştir. Mevcut çalışma için Cronbach alfa tutarlılık katsayısı .85 olarak bulunmuştur.

Kabul ve Eylem Formu - II (KEF-II): Kabul ve Eylem Formu - II, Hayes ve arkadaşları tarafindan (2004) Kabul ve Kararlılık Terapisi'nin temel boyutlarından birini oluşturan psikolojik katılığın kişiler arasındaki farklılıklarını ölçmek amacıyla geliştirilmiştir. Ölçek 7 madde ve tek boyuttan oluşmaktadır. Ölçekten alınan puan arttıkça psikolojik katılık ve yaşantısal kaçınma artmakta psikolojik esneklik azalmaktadır (Bond ve ark., 2011). Ölçeğin ülkemizdeki uyarlama çalışması Yavuz ve arkadaşları tarafından (2016) gerçekleştirilmiştir. Ölçeğin Cronbach alfa tutarlılık katsayısı .84 ve test tekrar test güvenirliği $\mathrm{r}=.85$ olarak bulunmuştur. Mevcut çalışma için Cronbach alfa tutarlılık katsayısı .92 'dir.

\section{İşlem}

Öncelikle çalışmanın yürütülebilmesi için İstanbul Medipol Üniversitesi etik kuruldan gerekli izinler alınmıştır (Karar No:65 Tarih:19.12.2019). Bu çalışma, araştırma ve yayın etiğine uygun olarak yürütülmüştür. Katılımcılara ölçekler internet üzerinden çevrimiçi olarak Google Formlar aracılığıyla yollanmıştır.

Katılımcılara araştırmaya katılmadan önce araştırma hakkında bilgi verildiği ve araştırmaya katılımın gönüllük esasına bağlı olduğunu içeren bilgilendirilmiş onam formu sunulmuştur. Katılımcıların onayı alındıktan sonra araştırma sorularına geçilmiştir. Ölçeklerin doldurulması yaklaşık 15 dakika sürmüştür.

\section{Bulgular}

\section{Korelasyon Analizine Ait Bulgular}

Katılımcıların zaman perspektifi alt boyutları, DZSP, kabul ve eylem formu ve psikolojik iyi oluş ölçeklerinden aldıkları puanların birbirleriyle ilişkilerini incelemek amaciyla Pearson Korelasyon Analizi yapılmıştır. Bulgular Tablo 1'de gösterilmiştir.

\section{Regresyon Analizi Bulguları}

Korelasyon analizinde psikolojik iyi oluş ile anlamlı ilişkiler gösteren değişkenler temelinde hiyerarşik regresyon analizi yürütülmüştür. Buna göre psikolojik iyi oluş düzeyi yordanan değişken; zaman perspektifi alt boyutları ve psikolojik katılık ise yordayıcı değişkenler olarak analize girmiştir. İlk adımda zaman perspektifi alt boyutları (olumlu geçmiş, olumsuz geçmiş, hazcı şimdi, kaderci şimdi ve gelecek) denkleme girdiğinde toplam varyansın \%48'ni açıkladığı görülmektedir $\left(R^{2}=\right.$ $.48, F(5,344)=62.40, p<.01)$. Ardından ikinci adımda denkleme giren psikolojik katılık ile açıklanan varyans 
Tablo 1. Değişkenlere Ait Pearson Korelasyon Analizi Bulguları

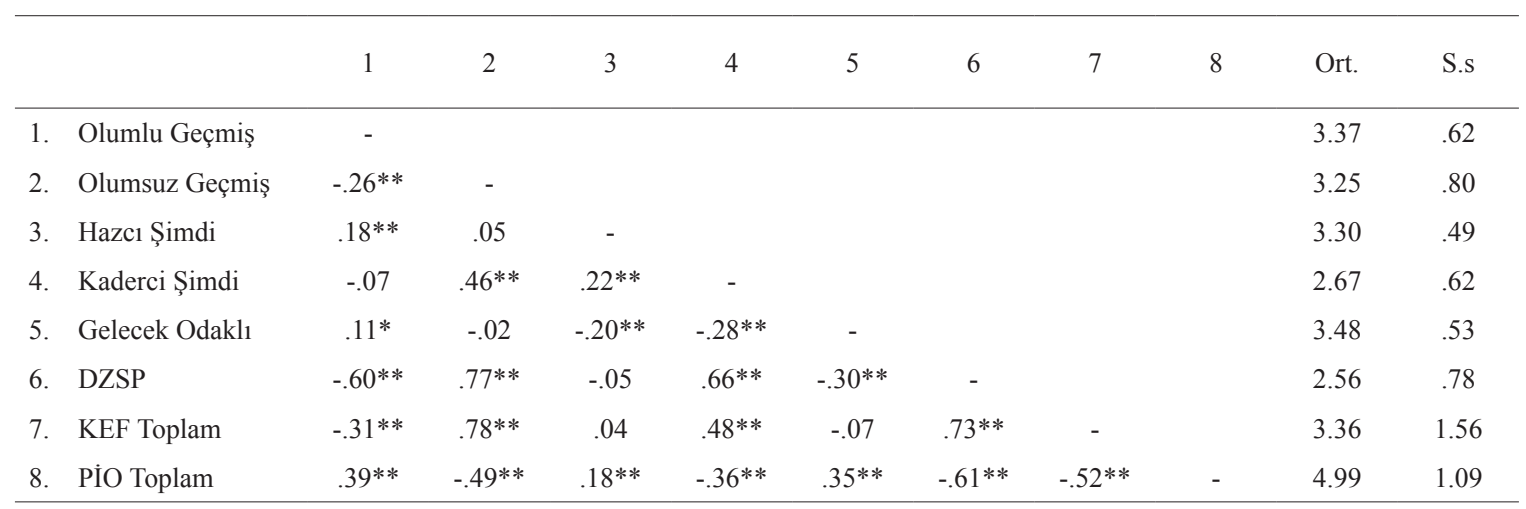

${ }^{*} p<.05 ; *^{*} p<.01$ DZSP: Dengeli Zaman Perspektifi Sapma Puanı, KEF: Kabul ve Eylem Formu -II, PİÖ: Psikolojik İyi Oluş Ölçeği

\%49'a yükselmiştir $\left(R^{2}=.49, F(6,343)=55.64, p<\right.$ .01). Bununla birlikte zaman perspektifi alt boyutlar1nın anlamlı etkisini sürdürdüğü görülmektedir. Bulgular Tablo 2'de gösterilmiştir.

\section{Aracı Değişken Analizi Bulguları}

DZSP ile psikolojik iyi oluş arasındaki ilişkide psikolojik esnekliğin/katılığın aracı rolü incelenmesi amacıyla verilere aracı değişken analizi uygulanmıştır. Bunun için Hayes (2013) tarafından geliştirilmiş olan PROCESS makro eklentisi kullanılmıştır. Aracılık etkisi, model 4 kullanılarak 1000 bootstrap örneklemi üzerinde incelenmiştir. Buna göre \%95'lik güven aralığında sıfır olmaması aracılık etkisinin anlamlı olduğuna işaret etmektedir (Hayes, 2013).

İlk olarak DZSP'nin aracı değişken olan psiko- lojik katılık düzeyini pozitif yönde anlamlı bir şekilde yordadığı görülmektedir $(B=1.46, S E=.07, \% 95 \mathrm{GA}$ $[1.3174,1.6071], p<.001)$. Aracı değişken olan psikolojik katılık düzeyinin ise psikolojik iyi oluş puanlarını negatif yönde anlamlı bir şekilde yordadığı görülmekte$\operatorname{dir}(B=-.12, S E=.04, \% 95$ GA [-.2039, -.0337], $p<$ $.05)$. Ayrıca DZSP'nin bağımlı değişken olan psikolojik iyi oluş puanları üzerinde toplam etkisinin anlamlı olduğu görülmektedir $(B=-.85, S E=.06, \% 95$ GA [-.9694, -.7334], $p<.001)$. DZSP psikolojik katılık ile birlikte denkleme girdiğinde ise psikolojik iyi oluş üzerindeki et-

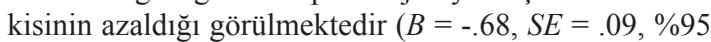
GA [-.8484, -.50ülke], $p<.001)$. Buna göre DZSP'nin psikolojik iyi oluş üzerinde psikolojik katılık aracılığıyla dolaylı etkisinin anlamlı olduğu görülmüştür ( $B=-17$, $S E=.06, \% 95$ GA [-3037, -0565], $p<.001)$. (Bkz. Şekil

Tablo 2. Regresyon Analizi Bulguları

\begin{tabular}{|c|c|c|c|c|c|c|c|c|c|}
\hline \multirow[b]{2}{*}{ Değişkenler } & & \multicolumn{4}{|c|}{ Model 1} & \multicolumn{4}{|c|}{ Model 2} \\
\hline & & $B$ & Std. Hata & $\beta$ & $t$ & $B$ & Std. Hata & $\beta$ & $t$ \\
\hline Olumsuz Geçmiş & & -.54 & .06 & -.39 & $-8.47 * *$ & -.33 & .09 & -.24 & $-3.78 * *$ \\
\hline Olumlu Geçmiş & & .34 & .07 & .20 & $4.65^{* *}$ & .30 & .07 & .17 & $4.00 * *$ \\
\hline Hazcı Şimdi & & .57 & .09 & .26 & $6.20 * *$ & .57 & .09 & .26 & $6.23^{* *}$ \\
\hline Kaderci Şimdi & & -.23 & .08 & -.13 & $-.2 .83 * *$ & -.17 & .08 & -.10 & $-.2 .04 *$ \\
\hline Gelecek Yönelimi & & .69 & .09 & .33 & $7.88^{* *}$ & .69 & .09 & .33 & $8.01 * *$ \\
\hline \multirow[t]{4}{*}{ KEF Toplam } & & & & & & -.16 & .05 & -.22 & $-3.46^{* *}$ \\
\hline & $R^{2}$ & & \multicolumn{3}{|c|}{.48} & \multicolumn{4}{|c|}{.49} \\
\hline & $F$ & & \multicolumn{3}{|c|}{$62.39 * *$} & \multicolumn{4}{|c|}{$55.64 * *$} \\
\hline & $d f$ & & \multicolumn{2}{|c|}{5.34} & & \multicolumn{4}{|c|}{6.34} \\
\hline
\end{tabular}

$* p<.05 ; * * p<.01$, KEF: Kabul ve Eylem Formu -II 


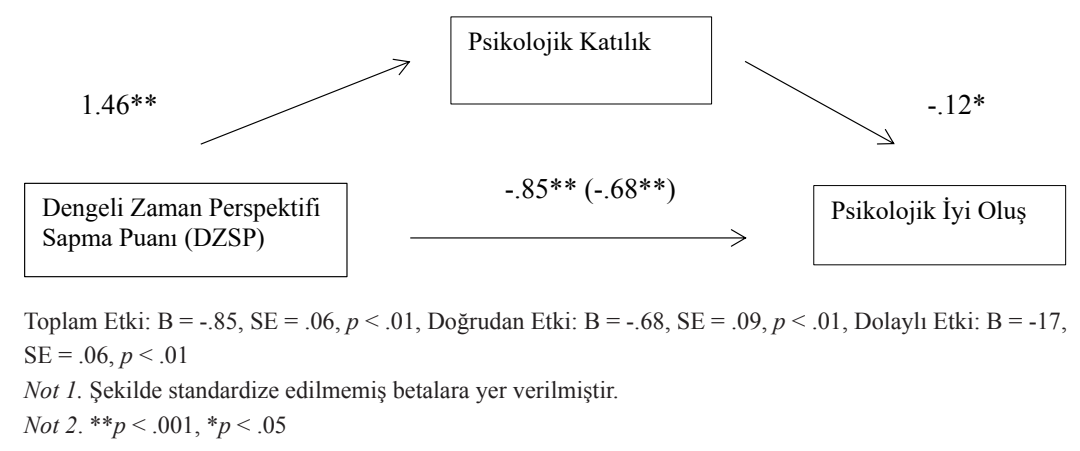

Şekil 1. DZSP ve psikolojik iyi oluş arasında psikolojik katılığın aracı rolü

\section{Tartışma}

$\mathrm{Bu}$ çalışma psikolojik iyi oluş üzerinde zaman perspektifi ve psikolojik esnekliğin yordayıcı gücünü araştırmak ve DZP ile psikolojik iyi oluş arasında psikolojik esnekliğin aracı rolünü incelemek amacıyla gerçekleştirilmiştir. Çalışmadan elde edilen bulgular zaman perspektifi beş alt boyutunun psikolojik iyi oluşu anlamlı düzeyde yordadığını ortaya koymaktadır. Olumlu geçmiş, şimdi hazcı ve gelecek zaman alt boyutlarının psikolojik iyi oluş üzerinde pozitif yordayıcı gücü olduğu görülürken olumsuz geçmiş ve kaderci şimdi alt boyutlarının psikolojik iyi oluş üzerinde negatif yordayıcı gücü olduğu saptanmıştır.

Elde edilen bulgular daha önceki araştırmalar ile tutarlılık göstermektedir. Buna göre mevcut çalışmada psikolojik iyi oluş düzeyini pozitif yönde yordayan olumlu geçmiş ve gelecek yönelimli olmanın yapılan önceki araştırmalarda psikolojik belirtiler ile negatif; psikolojik iyi oluş ve yaşam doyumu ile ise pozitif ilișkili olduğu görülmektedir (Boniwell ve ark., 2010; Pethel, Moist ve Baker, 2018; Sailer ve ark., 2014; Zimbardo ve Boyd, 1999). Webster ve arkadaşlarının (2014) zaman perspektifi üzerine yaptıkları çalışma sonuçlarına göre kişi geçmişi referans alarak, sorunlarla başa çıkmada başarılı olduğu zamanları hatırlayabilir ve zamanla olumlu yöndeki değişimleri ve gelişimleri görebilir. Ayrıca değer verdiği insanlarla ilgili hoş ve mutlu yaşantılar ile oluşturulmuş otobiyografik anılar kişilerin psikolojik, duygusal ve sosyal refahını artırabilir. Benzer şekilde kişinin gelecekteki hedefleri, elde edilebileceği başarıları hayal etmesi ve kişi için anlamlı başarıları öngörmesi, geleceğe dair olumlu bir yönelim ile zihinsel sağlığına katkıda bulunabilir. Ayrıca daha önce yapılan çalışmalarda da gelecek odaklı olmanın psikopatoloji ile negatif korelasyon içinde olduğu bulunmuştur (Wallace, 1956). Bu aktarılanlar 1şığında geçmişlerini daha olumlu ele alan, doğal ve anlık davranan, geleceğe dair hedefleri olan ve bu hedefler doğrultusunda hareket eden insanların psikolojik iyilik hallerinin de yüksek olduğu söylenebilir.

Tüm bunlarla beraber gelecek odaklı olmanın sadece hedeflere odaklanma ile birlikte andaki eğlenceyi kaçırmaya ve aile değerlerini yıpratmaya sebep olabileceği de iddia edilmiştir (Boniwell ve Zimbardo, 2003). Yapılan bir araştırmada gelecek yönelimi ile iyi oluş arasında ilişki bulunamamış ve araştırmacılar gelecekteki hedeflere odaklanmanın insanları şimdiki gerçeklikten uzaklaştıracağını aynı zamanda daha fazla endişe ve aciliyet hissi yaratabileceğini öne sürmüşlerdir (Drake ve ark., 2008). Başka bir çalışmada ise gelecek zaman yönelimi ve nevrotiklik arasında pozitif ilişki bulunmuştur (Dunkel ve Weber, 2010). Ancak mevcut araştırmada gelecek zaman yöneliminin psikolojik iyi oluş üzerindeki etkisi olumlu geçmiş zaman yöneliminden daha fazladır. Burada sosyo-kültürel değişkenlerin etkisinden söz edilebilir. Mevcut araştırma kolektivizmin ön planda olduğu bir kültür olan Türkiye'de yürütülmüştür (Hofstede, Hofstede ve Minkov, 2010). Kolektivizm bir aileye, bir gruba ya da organizasyona güçlü bağ $\operatorname{ll}_{1} \breve{g ̆}_{1}$ vurgular (Hofstede ve Bond, 1984). Bireyselliğin ön planda olduğu kültürlerde yapılan çalışmalarda kişinin gelecek zaman yönelimli olması kişiyi sosyal bağlardan koparabilecek güce sahip olabilirken; Türkiye örnekleminde kişinin sosyal bağlarını etkileyemediği ve kişinin gelecek zaman perspektifinin diğer bileşenleri olan sorumluluk duygusu, devamlılık gibi pozitif özelliklere yönelik bir etkiye sahip olduğu yorumu yapılabilir.

Elde edilen bulgulara göre olumsuz geçmiş ve kaderci şimdi zaman perspektiflerinin psikolojik iyi oluş üzerinde negatif yordayıcı gücü olduğu saptanmıştır. Zimbardo ve Boyd'un (1999) yaptıkları araştırmada da olumsuz geçmiş zaman ve kaderci şimdi perspektifi depresyon, anksiyete, düşük özsaygı ve öfke ile ilişsili bulunmuştur. Mevcut araştırmada olumsuz geçmiş zamanın psikolojik iyi oluşu, olumlu geçmiş zamandan daha güç- 
lü açıkladığı saptanmıştır. Bu bulgu psikolojik iyi oluş üzerinde olumsuz olayların olumlu olaylara göre daha güçlü etkisi olduğu bulgusunu desteklemektedir (Baumeister, Bratslavsky, Finkenauer ve Vohs, 2001). Bununla birlikte kaderci şimdi zaman perspektifi, psikolojik iyi oluşu negatif yönde açıklamakla birlikte en zayıf açıklayan değişken olmuştur. Yapılan bir araştırmada kaderci şimdi zaman perspektifinin psikolojik iyi oluş ile ilişkisi diğer perspektiflere oranla daha güçlüdür (Sailer ve ark., 2014). Bahsedilen araştırmanın elde ettiği kaderci şimdi perspektifi ile psikolojik iyi oluş arasındaki bu güçlü ilişki kaderci şimdi perspektifin kişinin kendi hayatı üzerine kontrol hissiyatını azaltması ve kişinin şimdide bulunduğu davranışların gelecekte bir şeyi değiştirmeyeceği düşüncesi ile beraber gelecek kaygısı taşıması ile açıklanmaktadır. Özetle kişi şimdiyi ve geleceği etkilemeyeceğini düşünürken gelecek hakkında kaygılanmaya devam eder (Drake ve ark., 2008). Bu sebeple kaderci şimdi zaman perspektifinin düşük iyi oluşla ilişkisi güçlüdür. Ancak mevcut araştırmada kaderci şimdinin psikolojik iyi oluş üzerindeki zayıf gücüne dair elde edilen bulgu örneklemin sosyo-kültürel yönü ile ilgili olabilir. Türk kültüründe kader inanc1, kontrolü tamamen birakmak olmamakla birlikte gelecek üzerine kaygıyı azaltmayı da ögütler. "Deveni sağlam kazığa bağla sonra tevekkül et" sözü kader inancına bir bakış açısını betimlemektedir (Zimbardo ve Boyd, 2018). Bu da bulgular arasındaki farklılığa bir açıklama olarak düşünülebilir.

Araştırma sonucunda elde edilen diğer bir bulgu ise psikolojik katılığın, psikolojik iyi oluşu negatif yönde yordamasıdır. Kabul ve Kararlılık Terapisi'nin temel kavramlarından biri olan psikolojik esneklik; farkında olma yeteneği, olumlu olumsuz deneyimlere açık olma ve değerler doğrultusunda hareket ile kişinin zorluklarla başa çıkarken daha etkili davranışlar sergileyeceğini söylemektedir. Böylece kişinin psikopatolojiden uzaklaşacağını, hayat kalitesinin artacağını ve nihai olarak iyi oluşunun yükseleceğini vurgulamaktadır (Harris, 2018). Yapılan araştırmalar esnekliği artıran müdahalelerin birçok psikopatoloji ve fizyolojik problemle başa çıkmada destek sağladığını göstermektedir (Bach ve Hayes, 2002; Bond ve Bunce, 2003; Dahl, Wilson ve Nilsson, 2004; Lappalainen ve ark., 2007). Psikolojik iyi oluş ve psikopatoloji birbirine bağımlı iki kavramdır (Keyes, 2007; Lamers, Westerhof, Glas ve Bohlmeijer, 2015). Psikolojik iyi oluşu arttıran etmenler (iyi sosyal ilişkiler, özerklik, uyum gibi) bir yandan psikolojik rahatsızlıklara karş1 da koruyucudur (Grant, Guille ve Sen, 2013). Buna göre psikolojik esnekliği arttıran müdahalelerin psikolojik iyi oluşu arttırdığ1 yorumu yapılabilir. Klinik olmayan örneklem ile yapılan bir araştırmada psikolojik esneklik ve bilinçli farkındalığın somatizasyon, kaygı, depresyon ve genel stresle negatif ilişkili olduğu görülmüştür (Masuda ve Tully, 2012). Psikolojik stres sorunu yaşayan katılımcılar ile yapılan psikolojik esnekliği arttırma müdahaleleri içeren grup terapisi çalışmaları sonucunda kişilerin duygusal ve psikolojik iyi oluşlarında ayrıca zihinsel sağlıklarında olumlu yönde artış gözlenmiştir (Fledderus, Bohlmeijer, Smit ve Westerhof, 2010). Buna göre teorik ve deneysel bilgiler 1şığında, mevcut çalışmada elde edilen psikolojik katılığın iyi oluşu negatif yönde yordaması bulgusu alanyazınla tutarlıdır.

Mevcut araştırmanın son bulgusuna göre, psikolojik esnekliğin/katılığın DZSP ve psikolojik iyi oluş ilişkisinde aracı rolü olduğudur. Buna göre DZSP diğer bir ifadeyle daha az DZP'ye sahip olmak psikolojik katılığ1 arttırmakta; bu da psikolojik iyi oluşu azaltmaktadır. DZP, bireylerin değişen çevresel koşullara uyumlarını en üst düzeye çıkarmalarına, kişisel hedeflerini ve değerlerini etkin bir şekilde gerçekleştirmelerine olanak tanıyan bir tür zamansal esneklik olarak da ele alınabilir (Stolarski, Zajenkowski, Jankowski ve Szymaniak, 2020). Boniwell ve Zimbardo (2015) bu kavramı pozitif psikoloji alanına tanıtırken; dış koşullara bağlı olarak uyarlanan, psikolojik ve fizyolojik sağlık açısından en uygun durumu sağlayan zihinsel süreçlerin bütünü olduğunu vurgulamışlardır. Pozitif psikolojinin "İyi bir yaşam nedir ve bunu nasıl elde ederiz?" sorusuna da DZP'ye ulaşmak ve dolayısıyla tatmin edici bir yaşam sürdürmek ile bir cevap önermektedir (Boniwell ve Zimbardo, 2003). Henüz yayımlanan bir meta analiz çalışmasında DZSP ve DZP üzerine yapılan araştırmalar incelenmiştir. $\mathrm{Bu}$ kapsamlı araştırmada DZSP ve DZP'nin iyi oluş üzerinde özellikle belirgin etkileri olan önemli bir uyum mekanizması olduğu sonucuna varılmıştır (Stolarski ve ark., 2020).

Mevcut çalışma bulguları, DZP ve psikolojik iyi oluş ilişkisinde psikolojik esnekliğin önemli bir rolü olduğuna işaret etmektedir. Zimbardo ve Boyd (2018) psikolojik iyi oluşu üst düzeye çıkarmak ve psikolojik sağlığa sahip olabilmek için zaman perspektifleri arasında esnek geçişler yapabilme gerekliliğini savunmuşlardır. Buna göre DZP'de bir zaman perspektifini tamamen ihmal etmek ya da tamamen birini kullanmak yerine perspektifler arasında içinde bulunan durum bağlamında esnek geçişler yapabilmek önemlidir (Zimbardo ve Boyd, 1999). Bu tarz bir esneklik ise mevcut çalışmanın da ortaya koyduğu üzere psikolojik esneklik düzeyini olumlu etkileyerek psikolojik iyi oluşu arttırmaya katk1 sunacaktır. Buna göre DZP'ye sahip olmanın olumlu yönde etkilediği psikolojik esneklik; farkındalık ve açıklıkla deneyimleri ele alma, anla temas etme ve değer odaklı davranışlar sergileme ile yaşam kalitesini yükseltmektedir (Harris, 2018). DZP'de olması gereken düşük olumsuz geçmiş zaman ve kaderci şimdi zaman perspektifi, psikolojik esnekliğin içerdiği deneyimleri açıklıkla ve kabulle ele almayı olumlu etkileyeceği şek- 
linde yorumlanabilir. Aynı zamanda DZP'de orta puan olması gereken şimdi hazcı perspektifi esneklikte anla teması; yüksek olması gereken gelecek zaman ve olumlu geçmiş zaman perspektifi ise değer odaklı davranışlarda bulunmayı etkileyeceği şeklinde açıklanabilir. Bunların yanı sıra psikolojik iyi oluşu arttırmak üzere psikolojik esnekliği kapsayan müdahalelerin de etkili olduğunu gösteren birçok çalışma vardır (Deledda ve ark., 2015; Marshall ve Brockman, 2016; Wersebe ve ark., 2018).

Ek olarak, Zimbardo zaman perspektifi kuramı üzerine oluşturduğu travma odaklı müdahale tekniklerinde kişinin düşüncesinin içeriğinden ziyade düşünme biçimi ve çerçevesi olan "zaman perspektifi" ile çalışmayı vurgulamış ve başarılı sonuçlar elde etmiştir (Zimbardo, Sword ve Sword, 2012). Psikolojik esneklik kavramında da benzer olarak "deneyimlere açık olmak", düşüncelerin içeriğine değer vermeden onları olduklar1 gibi kabul etmek ve gelip gitmelerine izin vermektir (Harris, 2018). Bu temelde zaman perspektifi üzerine çalışmak, düşüncelerin içeriğinden ziyade çerçeveleri üzerine çalışmak olacağından, esnekliğin de olumlu etkilenebileceği yorumu yapılabilir. $\mathrm{Bu}$ çıkarımlara göre kişinin DZP'ye ulaşması için yapılan müdahalelerle psikolojik esneklik arttırılabilir ve bu şekilde psikolojik iyi oluşu yükseltilebilir.

Alanyazında DZP ve psikolojik iyi oluşla ilgili birçok çalışma bulunurken bu ilişskide esnekliğin rolü ile ilgili bir çalışmaya rastlanmamıştır. Mevcut çalışmada deneysel verilerle esnekliğin aracı rolünün saptanmış olması zaman perspektifi kuramı ile tutarlıdır ve klinik düzlemde kullanılabilecek yararlı bir bilgidir.

Mevcut çalışmanın bazı sınırlılıkları bulunmaktadır. İlk olarak çalışmada öz bildirime dayalı ölçekler kullanılmıştır. Bundan dolayı öz bildirime dayalı ölçeklerin sınırlılıklarını içermektedir. Araştırmanın örnekleminin büyük çoğunluğu yüksek eğitim seviyesine sahip katılımcılardan oluşmaktadır. Kaderci şimdi ve gelecek zaman perspektifinin eğitim düzeyi ile oldukça ilişkili olduğu göz önüne alındığında elde edilen veriler yüksek eğitim düzeyi ile sınırlıdır. Araştırma örnekleminin yaş ortalaması 29.53'tür. Örneklem 18 yaş üstü ile sınırlıdır. Zaman perspektifi kuramında ideale yaklaşmak için bir öğrenme ve değişme sürecinden bahsedilmektedir. Beynin esnekliğinin (plastisitesinin) fazla olduğu dolayısıyla öğrenme ve değişimin daha hızlı olduğu ergenlik döneminde zaman perspektifi çalışmak değerli olabilir. Araştırma örnekleminin \%86.6'sının hayatlarını en çok geçirdikleri yer şehir ve büyükşehirdir. Öğrenilen zaman perspektifinin yaşanılan bölgenin özelliklerinden etkilendiği bilinmektedir. Dolayısıyla hayatlarının büyük kısmını köy ve kasaba gibi daha küçük yerleşim biriminde geçiren katılımcıların zaman perspektifleri daha kapsamlı incelenebilir
Zaman perspektifi evrensel bir kavram olmasiyla birlikte öğrenme süreci ile bağlantılıdır. Dolayısıyla kültür ile birlikte ele alınmalıdır. Türkiye' de konu henüz yeni yeni dikkat çekmiştir ve araştırma sayısı azdır. Ülkemizde yapılacak araştırmalar ile zaman perspektifinin daha iyi anlaşılacağı ve üzerine daha kapsamlı yorum yapılabileceği düşünülmektedir. 


\section{Kaynaklar}

Akirmak, U. (2019). The validity and reliability of the Zimbardo time perspective inventory in a Turkish sample. Current Psychology, (Gao 2011). doi:10.1007/s12144-019-0153-3

Andrews, F. M. (1974). Social Indicators of Perceived Life Quality. Social Indicators Research I içinde (ss. 279-299). D. Reidel Publishing Company. doi:10.1016/0149-7189(80)90013-0

Babür, S. (2014). Aristotales Fizik. (S. Babür, Ed.). Ankara: Yapı Kredi Yayınları.

Bach, P. ve Hayes, S. C. (2002). The use of acceptance and commitment therapy to prevent the rehospitalization of psychotic patients: A randomized controlled trial. Journal of Consulting and Clinical Psychology, 70(5), 1129-1139. doi:10.1037/0022006X.70.5.1129

Baumeister, R. F., Bratslavsky, E., Finkenauer, C. ve Vohs, K. D. (2001). Bad Is Stronger Than Good. Review of General Psychology, 5(4), 323-370. doi:10.1037/1089-2680.5.4.323

Bond, F. W. ve Bunce, D. (2003). The Role of Acceptance and Job Control in Mental Health, Job Satisfaction, and Work Performance. Journal of Applied Psychology, 88(6), 1057-1067. doi:10.1037/00219010.88.6.1057

Bond, F. W., Hayes, S. C., Baer, R. A., Carpenter, K. M., Guenole, N., Orcutt, H. K., ... Zettle, R. D. (2011). Preliminary Psychometric Properties of the Acceptance and Action Questionnaire-II: A Revised Measure of Psychological Inflexibility and Experiential Avoidance. Behavior Therapy, 42(4), 676-688. doi:10.1016/j.beth.2011.03.007

Boniwell, I. (2005). Can coaching strategies help experts share expertise? International Journal of Evidence Based Coaching and Mentoring, 3(2), 14. http://www.business.brookes.ac.uk/research/areas/ coaching\&mentoring/ adresinden erişildi.

Boniwell, I., Osin, E., Alex Linley, P. ve Ivanchenko, G. V. (2010). A question of balance: Time perspective and well-being in British and Russian samples. Journal of Positive Psychology, 5(1), 24-40. doi:10.1080/17439760903271181

Boniwell, I. ve Zimbardo, P. G. (2003). Time to Find the Right Balance. The Psychologist, 16:3, 129-131.

Boniwell, I. ve Zimbardo, P. G. (2015). Balancing Time Perspective in Pursuit of Optimal Functioning. Positive Psychology in Practice: Promoting Human Flourishing in Work, Health, Education, and Everyday Life: Second Edition, 223-236. doi:10.1002/9781118996874.ch13

Bryant, F. B. ve Veroff, J. (1982). The Structure of Psy- chological Well-being: A Sociohistorical Analysis. Journal of Personality and Social Psychology, 43(4), 653-673. doi:10.1037/0022-3514.43.4.653

Buccheri, R., Gesù, V. di ve Saniga, M. (2012). Studies on the structure of time: From Physics to Psycho(patho) logy. Springer Science \& Business Media.

Dahl, J., Wilson, K. G. ve Nilsson, A. (2004). Acceptance and commitment therapy and the treatment of persons at risk for long-term disability resulting from stress and pain symptoms: A preliminary randomized trial. Behavior Therapy, 35(4), 785-801. doi:10.1016/S0005-7894(04)80020-0

Deledda, G., Poli, S., Giansante, M., Zamboni, M., Turazza, M. ve Gori, S. (2015). Psychological well-being, acceptance and psychological flexibility, in breast cancer patients undergoing mastectomy or lumpectomy. Annals of Oncology, 26(Supplement 6), vi124. doi:10.1093/annonc/ mdv347.06

Diener, E., Wirtz, D., Biswas Diener, R., Tov, W., Kim Prieto, C., Choi, D. won ve Oishi, S. (2009). New Measures of Well-Being. Assessing Well-Being: The Collected Works of Ed Diener, içinde . Springer Science+Business Media. doi:10.1007/978-90-481-2354-4

Drake, L., Duncan, E., Sutherland, fi, Abernethy, C. ve Henry, C. (2008). Time Perspective and Correlates of Wellbeing. Time \& Society, 17(1), 47-61. doi:10.1177/0961463X07086304

Dunkel, C. S. ve Weber, J. L. (2010). Using three levels of personality to predict time perspective. Current Psychology, 29(2), 95-103. doi:10.1007/s12144010-9074-x

Fledderus, M., Bohlmeijer, E. T., Smit, F. ve Westerhof, G. J. (2010). Mental health promotion as a new goal in public mental health care: A randomized controlled trial of an intervention enhancing psychological flexibility. American Journal of Public Health, 100(12), 2372-2378. doi:10.2105/ AJPH.2010.196196

Ghorbani, M. ve Kazemi-zahrani, H. (2015). Psychological Well-Being and Creativity, (3), 20-23.

Grant, F., Guille, C. ve Sen, S. (2013). Well-Being and the Risk of Depression under Stress. PLoS ONE, 8(7), 1-6. doi:10.1371/journal.pone.0067395

Green S B. (1991). How Many Subjects Does It Take To Do A Regression Analysis. Multivariate Behavioral Research, 26(3), 499-510. doi:10.1207/ s15327906mbr2603

Harris, R. (2018). ACT'I Kolay Öğrenmek Illkeler ve Ötesi Iç̧in Hızlı Bir Başlangıç. Litera Yayıncılık.

Hayes, A. F. (2013). Introduction to mediation, moderation, and conditional process analysis: A regression-based approach. Guilford Press. 
Hayes, S. C., Luoma, J. B., Bond, F. W., Masuda, A ve Lillis, J. (2006). Acceptance and Commitment Therapy: Model, processes and outcomes. Behaviour Research and Therapy, 44(1), 1-25. doi:10.1016/j.brat.2005.06.006

Hayes, S. C., Strosahl, K. ve Wilson, K. G. (1999). Acceptance and Commitment Therapy: An experiential approach to behavior change. New York: Guilford Press.

Hayes, S. C., Strosahl, K., Wilson, K. G., Bissen, R. T., Polusny, M. A., Stewart, S. H., ... Karekla, M. (2004). Measuring experiential avoidance: a preliminary test of a working model steven $\mathrm{c}$. hayes kirk strosahl, 553-578.

Hofstede, G. ve Bond, M. H. (1984). Hofstede's culture dimensions: An Independent Validation Using Rokeach's Value Survey. Journal of Cross-Cultural Psychology, 15(4), 417-433. doi:10.1177/0022002184015004003

Hofstede, G., Hofstede, G. J. ve Minkov, M. (2010). Culture and Organizations. International Studies of Management \& Organization (C. 10). doi:10.1 080/00208825.1980.11656300

Holman, E. A. ve Zimbardo, P. G. (2009). The social language of time: The time perspective-social network connection. Basic and Applied Social Psychology, 31(2), 136-147. doi:10.1080/01973530902880415

James, W. (1890). The Principles of Psychology. New York: St Martin's Press.

Jankowski, K. S., Zajenkowski, M. ve Stolarski, M. (2020). What Are the Optimal Levels of Time Perspectives? Deviation from the Balanced Time Perspective-Revisited (DBTP-r). Psychologica Belgica, 60(1), 164-183. doi:10.5334/pb.487

Kaplan, G. A., Shema, S. J. ve Leite, C. M. A. (2008). Socioeconomic Determinants of Psychological Well-Being: The Role of Income, Income Change, and Income Sources During the Course of 29 Years. Annals of Epidemiology, 18(7), 531-537. doi:10.1016/j.annepidem.2008.03.006

Keyes, C. L. M. (2007). Promoting and Protecting Mental Health as Flourishing: A Complementary Strategy for Improving National Mental Health. American Psychologist, 62(2), 95-108. doi:10.1037/0003066X.62.2.95

Keyes, C. L. M., Shmotkin, D. ve Ryff, C. D. (2002). Optimizing well-being: The empirical encounter of two traditions. Journal of Personality and Social Psychology, 82(6), 1007-1022. doi:10.1037/00223514.82.6.1007

Kim, H. K. ve McKenry, P. C. (2002). The relationship between marriage and psychological well-being: A longitudinal analysis. Journal of Family Issues, 23(8), 885-911. doi:10.1177/019251302237296
Laghi, F., Baiocco, R., D’Alessio, M. ve Gurrieri, G. (2009). Suicidal ideation and time perspective in high school students. European Psychiatry, 24(1), 41-46. doi:10.1016/j.eurpsy.2008.08.006

Lamers, S. M. A., Westerhof, G. J., Glas, C. A. W. ve Bohlmeijer, E. T. (2015). The bidirectional relation between positive mental health and psychopathology in a longitudinal representative panel study. Journal of Positive Psychology, 10(6), 553-560. doi:10.1080/17439760.2015.1015156

Lappalainen, R., Lehtonen, T., Skarp, E., Taubert, E., Ojanen, M. ve Hayes, S. C. (2007). The Impact of CBT and ACT Models Using Psychology Trainee Therapists: A Preliminary Controlled Effectiveness Trial. Behavior Modification, 31(4), 488-511. doi:10.1177/0145445506298436

Lewin, K. (1951). Field Theory in Social Science: Selected Theoretical Papers. New York: Harper.

Luoma, J. B. ve Vilardaga, J. P. (2013). Improving Therapist Psychological Flexibility While Training Acceptance and Commitment Therapy: A Pilot Study. Cognitive Behaviour Therapy, 42(1), 1-8. doi:10.1 080/16506073.2012.701662

Lyubomirsky, S., King, L. ve Diener, E. (2005). The benefits of frequent positive affect: Does happiness lead to success? Psychological Bulletin, 131(6), 803-855. doi:10.1037/0033-2909.131.6.803

Marshall, E. J. ve Brockman, R. N. (2016). The relationships between psychological flexibility, self-compassion, and emotional well-being. Journal of Cognitive Psychotherapy, 30(1), 60-72. doi:10.1891/0889-8391.30.1.60

Masuda, A. ve Tully, E. C. (2012). The role of mindfulness and psychological flexibility in somatization, depression, anxiety, and general psychological distress in a nonclinical college sample. Journal of Evidence-Based Complementary and Alternative Medicine, 17(1), 66-71. doi:10.1177/2156587211423400

McKay, M. T., Worrell, F. C., Zivkovic, U., Temple, E., Mello, Z. R., Musil, B., ... Perry, J. L. (2019). A balanced time perspective: Is it an exercise in empiricism, and does it relate meaningfully to health and well-being outcomes? International Journal of Psychology, 54(6), 775-785. doi:10.1002/ ijop. 12530

Pethel, O., Moist, M. ve Baker, S. (2018). Time Perspective and Psychological Well-Being in Younger and Older Adults. Journal of Positive Psychology and Wellbeing, 2(1), 45-63. doi:10.1007/s10162-016-0594-4

Powers, M. B., Zum Vörde Sive Vörding, M. B. ve Emmelkamp, P. M. G. (2009). Acceptance and commitment therapy: A meta-analytic review. Psy- 
chotherapy and Psychosomatics, 78(2), 73-80. doi:10.1159/000190790

Ramaci, T., Bellini, D., Presti, G. ve Santisi, G. (2019). Psychological flexibility and mindfulness as predictors of individual outcomes in hospital health workers. Frontiers in Psychology, 10(JUN), 1-12. doi:10.3389/fpsyg.2019.01302

Räsänen, P., Lappalainen, P., Muotka, J., Tolvanen, A. ve Lappalainen, R. (2016). An online guided ACT intervention for enhancing the psychological wellbeing of university students: A randomized controlled clinical trial. Behaviour Research and Therapy, 78, 30-42. doi:10.1016/j.brat.2016.01.001

Ryff, C. D. (1989). Happiness is Everything, or Is It? Explorations on the Meaning of Psychological Well-Being. Journal of Personality and Social Psychology, 57(6), 1069-1081. doi:10.1037/00223514.57.6.1069

Sailer, U., Rosenberg, P., Nima, A. Al, Gamble, A., Gärling, T., Archer, T. ve Garcia, D. (2014). A happier and less sinister past, a more hedonistic and less fatalistic present and a more structured future: time perspective and well-being. PeerJ, 2, e303. doi: $10.7717 /$ peerj.303

Seema, R. ve Sircova, A. (2013). Mindfulness - a Time Perspective? Estonian Study. Baltic Journal of Psychology, 14(1999), 4-21.

Sircova, A., Van De Vijver, F. J. R., Osin, E., Milfont, T. L., Fieulaine, N., Kislali-Erginbilgic, A., ... Boyd, J. N. (2014). A global look at time: A 24-country study of the equivalence of the zimbardo time perspective inventory. SAGE Open, 4(1), 1-12. doi:10.1177/2158244013515686

Stock, W. A., Okun, M. A. ve Benin, M. (1986). Structure of Subjective Well-being Among the Elderly. Psychology and aging, 1(2), 91-102. doi:10.1037/0882-7974.1.2.91

Stolarski, M., Bitner, J. ve Zimbardo, P. G. (2011). Time perspective, emotional intelligence and discounting of delayed awards. Time \& Society, 20(3), 346363. doi:10.1177/0961463X11414296

Stolarski, M., Matthews, G., Postek, S., Zimbardo, P. G. ve Bitner, J. (2014). How We Feel is a Matter of Time: Relationships Between Time Perspectives and Mood. Journal of Happiness Studies, 15(4), 809-827. doi:10.1007/s10902-013-9450-y

Stolarski, M., Zajenkowski, M., Jankowski, K. S. ve Szymaniak, K. (2020). Deviation from the balanced time perspective: A systematic review of empirical relationships with psychological variables. Personality and Individual Differences, 156(May 2019), 109772. doi:10.1016/j. paid.2019.109772
Strathman, A., Gleicher, F., Boninger, D. S. ve Edwards, C. S. (1994). The Consideration of Future Consequences. Journal of personality and social psychology, 66(4), 742-752. http://journals. ohiolink.edu/ejc/article.cgi?issn $=00223514 \&$ issue $=$ v66i0004\&article $=742$ _tcofc adresinden erişildi.

Telef, B. B. (2013). Psikolojik İyi Oluş Ölçeği: Türkçeye Uyarlama, Geçerlik ve Güvenirlik Çalışması The Adaptation of Psychological Well-Being into Turkish: A Validity and Reliability Study. Journal of Education), 28(3), 374-384. doi:10.13140/ RG.2.1.2414.4480

Van Beek, W., Berghuis, H., Kerkhof, ad ve Beekman, A. (2011). Time perspective, personality and psychopathology: Zimbardo's time perspective inventory in psychiatry. Time \& Society, 20(3), 364-374. doi:10.1177/0961463X10373960

Wallace, M. (1956). Future time perspective in schizophrenia. Journal of Abnormal and Social Psychology, 52(2), 240-245. doi:10.1037/h0039899

Webster, J. D., Bohlmeijer, E. T. ve Westerhof, G. J. (2014). Time to flourish: The relationship of temporal perspective to well-being and wisdom across adulthood. Aging and Mental Health, 18(8), 10461056. doi:10.1080/13607863.2014.908458

Webster, J. D. ve Ma, X. (2013). A balanced time perspective in adulthood: Well-being and developmental effects. Canadian Journal on Aging, 32(4), 433-442. doi:10.1017/S0714980813000500

Wersebe, H., Lieb, R., Meyer, A. H., Hofer, P. ve Gloster, A. T. (2018). The link between stress, well-being, and psychological flexibility during an Acceptance and Commitment Therapy self-help intervention. International Journal of Clinical and Health Psychology, 18(1), 60-68. doi:10.1016/j. ijchp.2017.09.002

Yavuz, F. (2015). Kabul ve Kararlılık Terapisi (ACT) Genel Bir Bakış Acceptance and Commitment Therapy (ACT): An Overview. Turkiye Klinikleri J Psychiatry, 8(2), 21-27.

Yavuz, F., Ulusoy, S., Iskin, M., Esen, F. B., Burhan, H. S., Karadere, M. E. ve Yavuz, N. (2016). Turkish version of acceptance and action Questionnaire-II (AAQ-II): A reliability and validity analysis in clinical and non-clinical samples. Klinik Psikofarmakoloji Bulteni, 26(4), 397-408. doi:10.5455/ bcp. 20160223124107

Zhang, J. W. ve Howell, R. T. (2011). Do time perspectives predict unique variance in life satisfaction beyond personality traits? Personality and Individual Differences, 50(8), 1261-1266. doi:10.1016/j. paid.2011.02.021 
Zimbardo, P. ve Boyd, J. (2018). Zaman Paradoksu. (E. A. Öztoplar, Ed.) (1. bs.). İstanbul: Pegasus Yayınları.

Zimbardo, P. ve Boyd, J. N. (1999). Putting time in perspective: A valid, reliable individual-differences metric. Time Perspective Theory; Review, Research and Application: Essays in Honor of Philip G. Zimbardo, (September), 17-55. doi:10.1007/9783-319-07368-2_2

Zimbardo, P. G., Keough, K. A. ve Boyd, J. N. (1997). Present time perspective as a predictor of risky driving. Personality and Individual Differences, 23(6), 1007-1023. doi:10.1016/S0191-8869(97)00113-X

Zimbardo, P., Sword, R. ve Sword, R. (2012). The Time Cure. New York: Jossey Bass. 


\section{Summary Psychological Well-Being; Time Perspective and Psychological Flexibility}

\author{
Lütfiye Nur Akkoç Arabaci ${ }^{1}$ \\ Istanbul Medipol University
}

\author{
Gizem Cesur Soysal \\ Istanbul Medipol University
}

Time perspective is defined as dividing human experience into three temporal categories as past, present and future (Zimbardo, Keough \& Boyd, 1997). According to Zimbardo and Boyd (2018), the time perspective is created via judgments learned through experiences, culture, education, social class, religion, climate and other conditions. The routine time perspective becomes a habit and shows continuity. Thus, the person often prefers one of the time perspectives to the other without being aware of it. The preferred time perspective plays an important role in one's emotions, thoughts and behaviors (Zimbardo \& Boyd, 2018). Zimbardo and Boyd (1999) developed a valid and reliable scale of time perspective namely the Zimbardo Time Perspective Inventory (ZTPI). ZTPI consists of five sub-dimensions; positive-past, negative-past, present-hedonistic, present-fatalistic, and future. Positive-Past represents an emotional, nostalgic and pleasant attitude to the past. This dimension also emphasizes maintaining relationships with family and friends. Negative-Past dimension evaluates the past as negative and disturbing. While traumatic and negative events may cause this assessment, only the person's perspective or the interaction of the two conditions can reveal this orientation. Present-Hedonistic represents focusing on present pleasure and not dealing with future outcomes. Present-Fatalistic is the attitude that the future cannot be predicted and intervened. Future perspective considers goals and success. Those who own future perspective behave as planned as possible in the present moment. Research have shown that time perspective predicts depression, anger, anxiety, self-esteem and educational achievement (Zimbardo \& Boyd, 1999), social relationships (Holman \& Zimbardo, 2009), mood (Stolarski, Matthews, Postek, Zimbardo \& Bitner, 2014) and subjective well-being (Drake, Duncan, Sutherland, Abernethy \& Henry, 2008).

Flexible and optimal use of the time perspective is shaped based on values and needs (Boniwell \& Zimbar- do, 2015). This concept is named as the balanced time perspective (BTP). BTP defined as the mental ability to switch effectively among time perspectives depending on the requirements of the situation and personal resources (Zimbardo \& Boyd, 1999). BTP plays a role on people's well-being (Boniwell \& Zimbardo, 2015).

Psychological well-being can be defined based on the distinction between positive and negative emotions and life satisfaction (Andrews, 1974; Bryant \& Veroff, 1982; Stock, Okun \& Benin, 1986). Psychological well-being is related to emotions and life satisfaction (Ryff, 1989). Research has shown that psychological well-being is associated with more stable marriages (Kim \& McKenry, 2002), higher income (Kaplan, Shema \& Leite, 2008), more creative ideas (Ghorbani \& Kazemi-zahrani, 2015) and stronger immune system (Lyubomirsky, King \& Diener, 2005). Moreover there is a strong positive relationship between psychological well-being and the balanced time perspective (Jankowski, Zajenkowski \& Stolarski, 2020; McKay et al., 2019; Webster \& Ma, 2013). In order to reach BTP one's require flexible transitions between time perspectives. Therefore, psychological flexibility might have a mediating role between BTP and psychological well-being.

Psychological flexibility $\backslash$ inflexibility is considered on the basis of Acceptance and Commitment Therapy (ACT) in the current study (Hayes, Strosahl \& Wilson, 1999). Accordingly, psychological flexibility can be defined as being in contact with the present moment, being aware of the feelings, accept all sensations and thoughts, even if they are unpleasant. Psychological flexibility also includes acting in line with personal values (Ramaci, Bellini, Presti \& Santisi, 2019). Studies have shown that ACT applications has shown increased flexibility and improved psychological well-being (Hayes, Luoma, Bond, Masuda \& Lillis, 2006). A meta-analysis study also indicates that ACT is effective in the treatment of various clinical disorders such as anxiety, depression and

Address for Correspondence: M.A. Lütfiye Nur Akkoç Arabacı, Istanbul Medipol University, Humanities and Social Sciences, Department of Psychology, Campus of Kavacik, Beykoz / Istanbul

E-mail: 1.nur.akkoc@gmail.com 
post-traumatic stress disorder (Powers, Zum Vörde Sive Vörding \& Emmelkamp, 2009).

In the light of these, the present study aims to investigate the role of psychological flexibility on the relationship between time perspective and psychological well-being.

\section{Method}

\section{Participants}

The sample consisted of 350 people who are 18 years of age and over. The average age was 29.53 (SD $=6.96$ ) years.

\section{Measurements}

Demographic Information Form. This form was designated by the researchers to collect social-demographic information about the participants.

Zimbardo Time Perspective Inventory (ZTPI). ZTPI was used to evaluate participants' time perspectives. The questionnaire is developed by Zimbardo and Boyd (1999) consists of 56 questions and five sub-dimensions. The Turkish validity and reliability study of the scale was conducted by Akırmak (2019) and it was found that the Cronbach's alpha for the sub-dimensions was between .68 and .84 . In the current study, Cronbach alpha was between .87 and .71

Flourishing Scale. Flourishing Scale is developed by Diener and colleagues (2009) which includes themes such as meaningful life, positive relationships, adequate self-perception, and having a goal. Adapted to Turkish culture by Telef (2013) and Cronbach alpha was found as .80. In the current study, Cronbach's alpha was .85.

Acceptance and Action Questionnaire-II (AAQ II). was developed by Hayes et al. (2004) to measure psychological inflexibility. Turkish adaptation was conducted by Yavuz et al. (2016) and Cronbach alpha was found as .84. In the current study, Cronbach's alpha was .92 .

\section{Procedure}

Ethical approval for this study was obtained from Istanbul Medipol University. Written informed consent was obtained from all subjects before the research. Data was collected from 350 participants using an online survey with a random sampling method.

\section{Results}

\section{Correlation Analysis}

The result of correlation analysis indicated that there was a significant positive correlation past positive $(r=.39, p<.01)$, hedonist present $(r=.18, p<.01)$ and future $(r=.35, p<.01)$ with psychological well-being. Increase in the psychological well-being was related with decrease in the past negative $(r=-.49, p<.01)$, present fatalistic $(r=-36, p<.01)$ and deviation from the balanced time perspective $(r=-.61, p<.01)$. Moreover, a significant negative correlation among psychological well-being $(r=-.52, p<.01)$ and psychological inflexibility were also noted.

\section{Regression Analysis}

A two-step hierarchical regression analysis was conduct to assess the predictors of psychological well-being. In the first step, all time perspectives were significant predictors of psychological well-being. This model explains the $48 \%$ of total variance $\left(R^{2}=.48, F(5\right.$, $344)=62.40, p<.01)$. In second step AAQ-II were entered to the equation. The results showed that AAQ-II was a significant predictor of psychological well-being. Besides, all time perspectives were still significant predictors in the final model. The last model's explained variance was $.49\left(R^{2}=.49, F(6,343)=55.64, p<.01\right)$.

\section{Mediation Analysis}

Mediation analyses were conducted using the PROCESS macro to assess indirectly effect of the psychological inflexibility level. The results show that the indirect effect of deviations from a balanced time perspective on psychological well-being through psychological inflexibility was significant $(B=-17, S E=.06$, $\% 95$ GA [-3037, -0565], $p<.001)$.

\section{Discussion}

Current study aimed to investigate the mediator role of psychological flexibility on the relationship between time perspective and psychological well-being. Second, this study examined the predictor role of the time perspective on psychological well-being. Accordingly the regression analysis results revealed that five profiles of time perspective predicted significantly psychological well-being. Considering past positively, benefiting from the pleasures of the present and acting with the responsibility for the future increases the psychological well-being. However, all negative schemes against the past and fate-oriented judgments decrease psychological well-being.

The mediator analysis result indicated that psychological flexibility plays a mediator role in the relationship between balanced time perspective (BTP) and psychological well-being. Accordingly, BTP affects psychological flexibility positively and psychological flexibility also increases well-being positively. Zimbardo and Boyd (2018) consider that a flexible transition between 
time perspectives is essential to maximize psychological well-being and psychological health. In a BTP, flexible transitions should be within the context of the situation, rather than completely neglecting a time perspective or using one (Zimbardo \& Boyd, 1999). This kind of flexibility will contribute to increasing psychological well-being by increasing the level of psychological flexibility, as this study indicated.

Psychological flexibility consists of being fully aware of experiences (emotions, sensations, and thoughts), welcoming them with openness and acceptance; including the undesired ones, being in contact with the present moment and expressing value-oriented behaviors (Harris, 2018). Therefore, low Past-Negative and Present-Fatalism in BTP can be interpreted as a positive effect on being aware of experiences and welcoming with openness and acceptance. Medium-high score of Present-Hedonistic in BTP can affect the contact with the present moment. High Past-Positive and Future in BTP, might have a positive effect value-oriented behaviours. Also, many studies showed that interventions involving psychological flexibility are effective to increase psychological well-being (Deledda et al., 2015; Marshall \& Brockman, 2016; Wersebe et al., 2018). Besides, researchers have emphasized the way of thinking rather than the content of thoughts in the time perspective theory and interventions (Zimbardo, Sword $\&$ Sword, 2012). Similarly, "being fully aware of experiences welcoming them with openness and acceptance" is to accept thoughts as they are and without allowing to assess the content of the thoughts (Harris, 2018) as in the case of psychological flexibility. On this basis, it can be interpreted that working on time perspective suggest working on the way of thinking rather than the content of thoughts. Thus, time perspective affects psychological flexibility positively. According to these conclusions, the psychological flexibility can be increased by interventions to achieve a balanced time perspective in turn the psychological well-being will be also increased.

Consequently, this study examined the mediator role of psychological flexibility on the relationship between time perspective and psychological well-being. While in the literature there are many studies on balanced time perspective and psychological well-being, there is no study on the role of psychological flexibility in this relationship. The results are consistent with the time perspective theory and they are useful for clinical practice. 